\title{
Perioperative outcomes with sutureless versus stented biological aortic valves in elderly persons
}

\author{
Jessica Forcillo, MD, MSc, Denis Bouchard, MD, PhD, Anthony Nguyen, MD, MSc, \\ Louis Perrault, MD, PhD, Raymond Cartier, MD, Michel Pellerin, MD, MSc, Philippe Demers, MD, MSc, \\ Louis Mathieu Stevens, MD, PhD, and Michel Carrier, MD, MBA
}

\begin{abstract}
Objectives: Sutureless aortic valves are deemed suitable for patients considered at high risk for surgery. The objective of this study is to evaluate the perioperative results of implanting a sutureless valve in elderly persons, compared with a stented biological valve in the aortic position.

Methods: Between 2011 and 2015, 76 patients underwent aortic valve replacement with the Perceval prosthesis (Sorin Group, Saluggia, Italy). The group was compared with 319 consecutive patients who received aortic valve replacement with the stented valve in that same period.
\end{abstract}

Results: The mean age of patients was $83 \pm 2$ years in the Perceval group and $83 \pm 3$ years in the stented valve group $(P=.3)$. Preoperative demographics were similar in both groups. Median cardiopulmonary bypass and crossclamp times were lower in the Perceval group than in the stented valve group $(P<.001)$. Mortality was $5 \%$ in the Perceval group and $6 \%$ in the stented valve group $(P=.8)$. There were more pacemaker implantations in the Perceval group than in the stented valve group $(17 \%$ vs $8 \% ; P=.02)$. A subgroup analysis of patients who underwent aortic valve replacement and concomitant procedures showed the same results as the entire cohort.

Conclusions: Aortic valve replacement with a sutureless prosthesis resulted in shorter aortic crossclamp and bypass times compared with a stented biological prosthesis. The reduced cardiopulmonary bypass and aortic crossclamp times obtained using the Perceval prosthesis did not translate into perioperative gains in our population of elderly patients. (J Thorac Cardiovasc Surg 2016;151:1629-36)

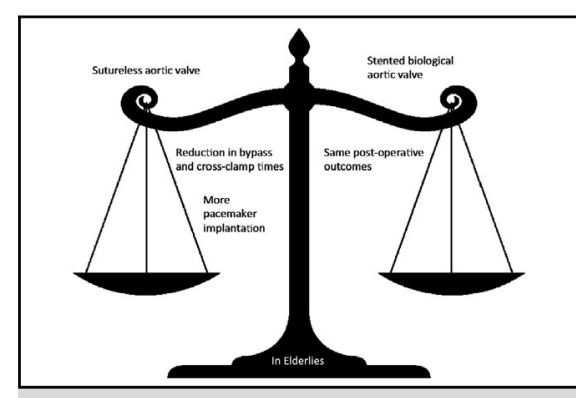

Sutureless aortic valve versus stented biological aortic valve.

\section{Central Message}

Sutureless aortic valve prosthesis decreases cardiopulmonary bypass time in elderly persons undergoing AVR with or without CABG surgery.

\section{Perspective}

This article evaluates perioperative outcomes of implanting these valves versus a stented biological valve in elderly persons. Despite a decrease in bypass and crossclamp times, we did not find a difference between groups in terms of mortality and morbidities, but we raised a flag regarding postoperative implantation of a pacemaker for third-degree AV block with the use of sutureless valves.

See Editorial Commentary page 1637.
Aging of the population is an established fact. Doctors are regularly confronted by the clinical challenges of treating older patients with advanced cardiovascular diseases and multiple comorbidities. With escalating healthcare costs, it has become imperative to examine the outcomes of patients undergoing surgical interventions in older age groups. In 8 different studies including

\footnotetext{
From the Department of Cardiac Surgery, Montreal Heart Institute and Université de Montréal, Montreal, Canada.

Received for publication June 5, 2015; revisions received Dec 7, 2015; accepted for publication Dec 29, 2015; available ahead of print Feb 17, 2016.

Address for reprints: Michel Carrier, MD, MBA, Division of Cardiac Surgery, Montreal Heart Institute, 5000 Belanger St, Montreal, QC H1T 1C8, Canada (E-mail: michel.carrier@icm-mhi.org).

$0022-5223 / \$ 36.00$

Copyright (C) 2016 by The American Association for Thoracic Surgery

http://dx.doi.org/10.1016/j.jtcvs.2015.12.056
}

1295 patients undergoing coronary artery bypass grafting $(\mathrm{CABG})$ and valve replacement procedures, it has been shown that cardiac surgery, in carefully selected octogenarians and nonagenarians, is justified and can be performed with acceptable results. However, increased mortality and morbidities, as well as increase length of stay, are associated with this age group. ${ }^{1-3}$ In this aging population, we find patients with multiple comorbidities and calcified ascending aorta and root. The actual challenge of our surgical specialty is to find strategies

Scanning this QR code will take you to the article title page.

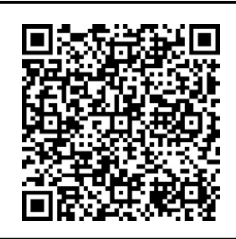




$$
\begin{array}{ll}
\text { Abbreviations and Acronyms } \\
\begin{array}{ll}
\text { AV } & =\text { atrioventricular } \\
\text { AVR } & =\text { aortic valve replacement } \\
\text { CABG } & =\text { coronary artery bypass grafting } \\
\text { euroSCORE }= & \text { European System for Cardiac } \\
& \text { Operative Risk Evaluation } \\
\text { ICU } & =\text { intensive care unit } \\
\text { IQR } & =\text { interquartile range } \\
\text { STS } & =\text { Society of Thoracic Surgeons } \\
\text { SV } & =\text { stented valve } \\
\text { TAVI } & =\text { transcatheter aortic valve } \\
& \text { implantation }
\end{array}
\end{array}
$$

to minimize the burden of an open cardiac surgery. Strategies aimed at reducing bypass and crossclamp times may reduce systemic inflammatory reaction and lead to decreased postoperative morbidities. Minimally invasive techniques also have been claimed to produce similar benefits.

Sutureless aortic valves have been introduced in practice in Europe since 2007 and in North America since 2009. This new technology is deemed suitable for patients who are considered at high risk for surgery. The Perceval S (Sorin Group, Saluggia, Italy) prosthesis is made of a trileaflet bovine pericardial valve mounted on an expandable metal frame of nitinol (equiatomic alloy of nickel and titanium). The Perceval $\mathrm{S}$ prosthesis has the special feature that it does not have to be surgically sutured into the implant site because this function is performed by the armature, which, along with supporting the valve edges, adapts itself to the aortic root to which it is anchored.

In high-risk patients aged more than 70 years, Santarpino and colleagues ${ }^{4}$ showed that implantation of the sutureless aortic valve Perceval reduced not only the operative time but also the number of transfusions received, intubation time, and length of stay in the intensive care unit (ICU). ${ }^{4}$ That same group published their 1-year results showing good hemodynamic profile and mean New York Heart Association functional class of $1 .^{5}$ Other groups have duplicated their results. 6,7

The sutureless prosthesis could be an interesting option in elderly persons with small aortic roots. A study by Shrestha and colleagues ${ }^{8}$ showed that the implantation of the Perceval valve compared with a standard stented aortic valve prosthesis in patients aged more than 75 years resulted in no annulus enlargement in the Perceval group versus 2 cases of annulus enlargement in the stented valve (SV) group. All patients enrolled in this study had an annular size less than $22 \mathrm{~mm}$. Some $24 \%$ of patients had a small Perceval valve, and $76 \%$ of patients had a medium Perceval valve. Perioperative and 5-year results showed no difference in mortality between groups. ${ }^{8}$ As in the case of $\mathrm{SVs}$, the Perceval valve offers greater effective orifice area for any given size. ${ }^{9}$

Sutureless valves have been used during minimally invasive surgery because of their short implantation time and ease of use. ${ }^{9}$ The safety of the valve has been demonstrated in various studies, especially in European centers, because of their longer experience with the sutureless prosthesis. $^{7}$

The objective of the present study is to evaluate the perioperative results of implanting a sutureless valve, the Perceval S, compared with a biological aortic SV in elderly patients.

\section{MATERIALS AND METHODS}

Between 2011 and 2015, 76 patients aged 80 years or more underwent aortic valve replacement (AVR) with the Perceval prosthesis and 319 patients received a biological SV. We included all consecutive patients aged more than 79 years who had an isolated AVR procedure or an AVR and CABG. Other concomitant procedures, including aortic annulus enlargement, dilatation of the ascending aorta, and redo surgery, were excluded. The study was approved by the Ethics Committees of the Montreal Heart Institute, the Hôpital Sacré-Coeur de Montréal, and the Centre Hospitalier de l'Université de Montréal. All surgeons who participated in this study are part of the Université de Montréal Cardiac Surgery Division. Those surgeons operated at the 3 different sites mentioned previously.

Valves were implanted in patients according to standard surgical techniques. The valves used in the standard conventional AVR group were the Carpentier-Edwards Perimount (Edwards Lifesciences, Irvine, Calif), Carpentier-Edwards Magna Ease (Edwards Lifesciences), Medtronic Mosaic (Medtronic Inc, Minneapolis, Minn), Sorin Mitroflow (Sorin Group), St Jude Biocor porcine (St Jude Medical, St Paul, Minn), and St Jude Epic standard and Supra (St Jude Medical). The surgical approaches included full and mini-sternotomy and mini-thoracotomy for both types of valves.

The surgical technique used to implant the Perceval valve has been well described in the literature. ${ }^{7}$ In brief, the native valve is excised, the annulus is decalcified, and 3 sutures of Prolene are placed at the nadir of each sinus. The prosthesis of the proper size is deployed in the aortic annulus. A balloon is inflated inside the prosthesis at 4 atm during 30 seconds. The aortotomy is closed in a standard fashion. The stented biological prostheses were implanted according to the standard surgical technique.

When the Perceval $\mathrm{S}$ is used for a combined procedure (eg, AVR + CABG), the ascending aorta is cannulated close to the aortic arch to leave room for proper placement of the proximal saphenous vein graft anastomoses on the ascending aorta. The aortotomy also is performed higher to allow the stented cage of the Perceval valve to abut properly on the aortic wall. After deployment of the sutureless valve, the heart is gently mobilized to avoid prosthesis dislodgement and embolization.

Outcomes of interest were recorded according to the Society of Thoracic Surgeons (STS) Guidelines for Reporting Mortality and Morbidity after Cardiac Valve Interventions Guidelines. ${ }^{10}$ The primary outcome of interest was perioperative (within 30 days) mortality. Secondary outcomes of interest were perioperative course and complications, such as reexploration for bleeding, myocardial infarction, ICU length of stay, intubation time, hospitalization time, number of red blood cell transfusions, renal failure requiring dialysis or continuous venovenous hemofiltration, complete or partial atrioventricular (AV) bloc 
requiring pacemaker implantation, atrial fibrillation, multiple organ failure, septicemia, and thromboembolic events (stroke, transient ischemic attack, and noncerebral embolic events). These outcomes were analyzed in the entire cohort of patients. A similar analysis was performed in the AVR and CABG subgroup to see if there was an advantage of using the Perceval valve in patients with a concomitant procedure. As an additional effort to control for systematic differences between patients receiving a Perceval valve and patients receiving an SV, a propensity score was constructed.

\section{Statistical Analysis}

Data were expressed as mean \pm standard deviation or median (interquartile range [IQR]) for continuous variables, as appropriate, and categoric variables were expressed as a number (percentage). Brackets were used to present IQR as a measure of dispersion for median for variables with non-normal distributions. Differences between continuous variables were analyzed using Student $t$ tests, and categoric variables were analyzed with chi-square tests.

Each patient who received a Perceval valve was propensity score matched to 1 patient receiving a standard AVR, using a 1:2 matching macro based on greedy algorithms. Variables included in the multivariable logistic regression model estimating the propensity score included female gender, estimated glomerular filtration rate (using the Chronic Kidney Disease Epidemiology Collaboration formulae), presence of pulmonary hypertension, and aortic prosthesis implant size (categorized in 4 groups). ${ }^{11}$ Patient characteristics before and after matching were compared using standardized differences. For 30-day mortality and hospital morbidity, logit-link generalized estimating equations were used accounting for matching. Likewise, identity-link normal-distribution generalized estimating equations were used for log-transformed cardiopulmonary bypass time, crossclamp time, chest tube drainage, number of transfusions, and length of stay. All statistical analyses were performed using SAS release 9.3 (SAS Institute Inc, Cary, NC).

\section{RESULTS}

\section{Entire Perceval Group Compared With Standard Prosthesis Group}

The mean age of patients was $83 \pm 2$ years in the Perceval group and $83 \pm 3$ years in the $\mathrm{SV}$ group $(P=.3)$ (Table 1$)$. Preoperative European System for Cardiac Operative Risk Evaluation (euroSCORE) II was 4.4\% (IQR, 3.0\%-8.7\%) in the Perceval group and $4.5 \%$ (IQR, $2.9 \%-7.8 \%$ ) in the

TABLE 1. Preoperative parameters for the entire cohort of patients

\begin{tabular}{|c|c|c|c|}
\hline Parameters & $\begin{array}{l}\text { Sutureless valve } \\
\qquad(\mathbf{N}=76)\end{array}$ & $\begin{array}{l}\text { Stented biological AVR } \\
\qquad(\mathrm{N}=319)\end{array}$ & $P$ value \\
\hline Age, y & $83 \pm 2$ & $83 \pm 3$ & .315 \\
\hline Sex (female) & $37(49 \%)$ & $149(47 \%)$ & .756 \\
\hline $\mathrm{BMI} / \mathrm{BSA}, \mathrm{kg} / \mathrm{m}^{2}, \mathrm{~m}^{2}$ & $28 \pm 4 / 2 \pm 0$ & $27 \pm 5 / 2 \pm 0$ & $.094 / .451$ \\
\hline euroSCORE $2(\%)$ & $4.4(3.0-8.7)$ & $4.5(2.9-7.8)$ & .710 \\
\hline STS predicted mortality ( $\%)$ & $5.7(4.3-8.6)$ & $5.6(4.2-7.8)$ & .588 \\
\hline Emergency/urgent surgery (\%) & $17(22)$ & $88(28)$ & .328 \\
\hline Preoperative LVEF (\%) & $58 \pm 9$ & $58 \pm 10$ & .702 \\
\hline Preoperative mean gradient ( $\mathrm{mm} \mathrm{Hg}$ ) & $47 \pm 16$ & $51 \pm 19$ & .210 \\
\hline Procedures & & & .037 \\
\hline Isolated aortic replacement & $43(57 \%)$ & $151(47 \%)$ & \\
\hline Aortic replacement and $\mathrm{CABG}$ & $32(42 \%)$ & $168(53 \%)$ & \\
\hline \multicolumn{4}{|l|}{ Surgical indication } \\
\hline Severe aortic stenosis & $69(91 \%)$ & $279(87 \%)$ & .421 \\
\hline Severe aortic regurgitation & $7(9 \%)$ & $40(13 \%)$ & .230 \\
\hline NYHA & & & .260 \\
\hline $\mathrm{I}$ & $18(24 \%)$ & $48(15 \%)$ & \\
\hline II & $25(33 \%)$ & $126(39 \%)$ & \\
\hline III & $30(39 \%)$ & $125(39 \%)$ & \\
\hline IV & $3(4 \%)$ & $20(6 \%)$ & \\
\hline \multicolumn{4}{|l|}{ Surgical approaches } \\
\hline Minimally invasive approaches & $14(18 \%)$ & $24(8 \%)$ & .034 \\
\hline Sternotomy & $62(82 \%)$ & $295(92 \%)$ & \\
\hline Aortic prosthesis & & & $<.001$ \\
\hline Carpentier-Edwards Perimount (Edwards Lifesciences, Irvine, Calif) & $0(0 \%)$ & $30(9 \%)$ & \\
\hline Carpentier-Edwards Magna Ease (Edwards Lifesciences, Irvine, Calif) & $0(0 \%)$ & $145(46 \%)$ & \\
\hline Medtronic Mosaic (Medtronic Inc, Minneapolis, Minn) & $0(0 \%)$ & $8(3 \%)$ & \\
\hline Pericardial Mitroflow (Sorin Group, Saluggia, Italy) & $0(0 \%)$ & $108(34 \%)$ & \\
\hline St Jude Biocor (St Jude Medical, St Paul, Minn) & $0(0 \%)$ & $9(3 \%)$ & \\
\hline St Jude Epic Standard (St Jude Medical) & $0(0 \%)$ & $2(1 \%)$ & \\
\hline St Jude Epic Supra (St Jude Medical) & $0(0 \%)$ & $13(4 \%)$ & \\
\hline Others & $76(100 \%)$ & $3(1 \%)$ & \\
\hline
\end{tabular}

$A V R$, Aortic valve replacement; BMI, body mass index; BSA, body surface area; euroSCORE, European System for Cardiac Operative Risk Evaluation; STS, Society of Thoracic Surgeons; $L V E F$, left ventricular ejection fraction; $C A B G$, coronary artery bypass grafting; NYHA, New York Heart Association. 
TABLE 2. Prosthetic aortic valve dimensions

\begin{tabular}{lccc}
\hline \multicolumn{1}{c}{ Size } & $\begin{array}{c}\text { Sutureless valve } \\
(\mathbf{N}=\mathbf{7 6})\end{array}$ & Size $(\mathbf{m m})$ & $\begin{array}{c}\text { Stented } \\
\text { biological AVR } \\
(\mathbf{N}=\mathbf{3 1 9})\end{array}$ \\
\hline Small & $14(18 \%)$ & 19 & $35(11 \%)$ \\
Medium & $23(30 \%)$ & 21 & $93(29 \%)$ \\
Large & $31(40 \%)$ & 23 & $104(33 \%)$ \\
Extra large & $8(11 \%)$ & 25 & $65(20 \%)$ \\
& & 27 & $21(7 \%)$ \\
& & 29 & $1(0 \%)$ \\
\hline
\end{tabular}

$\overline{A V R}$, Aortic valve replacement.

SV group $(P=.7)$. The STS score for predicted mortality was $5.7 \%$ (IQR, $4.3 \%-8.6 \%$ ) in the Perceval group and $5.6 \%$ (IQR, $4.2 \%-7.8 \%$ ) in the SV group $(P=.6)$. Mean preoperative left ventricular ejection fraction was normal in both groups: $58 \% \pm 9 \%$ and $58 \% \pm 10 \%$, respectively $(P=.7)$. Mean preoperative aortic gradients also were similar: $47 \pm 16 \mathrm{~mm} \mathrm{Hg}$ and $51 \pm 19 \mathrm{~mm} \mathrm{Hg}$, respectively $(P=.18)$ (Table 1). Prosthetic aortic valve dimensions are shown in Table 2.

Mean cardiopulmonary bypass times and crossclamp times were lower in the Perceval group than in the SV group: $64 \pm 21$ minutes versus $93 \pm 37$ minutes and $50 \pm 22$ minutes versus $74 \pm 29$ minutes, respectively $(P<.001)$ (Table 3).

There were 4 perioperative deaths $(5 \%)$ in the Perceval group and 19 deaths $(6 \%)$ in the SV group $(P=.8)$. Pacemaker implantation was more prevalent in the Perceval group ( $17 \%$ vs $8 \% ; P=.02)$. There was no significant difference between the 2 groups in the incidence of any reoperation and tamponade $(P=.9)$, myocardial infarction $(P=1.0)$, acute kidney injury $(P=.2)$ renal failure requiring dialysis $(P=.2)$, any cerebrovascular accident $(P=.9)$, atrial fibrillation $(P=.3)$, and septicemia $(P=.1)$. ICU length of stay $(P=.5)$, ICU reintubation $(P=.7)$, readmission to ICU or from home $(P=.4)$, number of red blood cell transfusions $(P=.8)$, and hospitalization time $(P=.5)$ also were similar between the 2 groups. There were no paravalvular leaks or patient-prosthesis mismatch in the 2 groups (Table 3 ).

Seven patients (10\%) had complete AV block, and 6 patients had a low-grade AV block and new left bundle branch block after Perceval deployment. Four patients had preoperative atrial fibrillation, 3 patients had a first-degree AV block, 1 patient had a right bundle branch block, and 1 patient had a first-degree AV block and left bundle branch block.

Table 4 shows the preoperative and postoperative mean echocardiography gradients and aortic valve areas. There were no significant differences between groups (Table 4). The postprocedure mean gradient for small Perceval prosthesis was $16 \pm 7 \mathrm{~mm} \mathrm{Hg}$ with a mean aortic area of
TABLE 3. Perioperative outcomes of the entire cohort of patients

\begin{tabular}{|c|c|c|c|}
\hline Outcomes & $\begin{array}{c}\text { Sutureless } \\
\text { valve } \\
(\mathbf{N}=76) \\
\end{array}$ & $\begin{array}{c}\text { Stented } \\
\text { biological AVR } \\
(\mathbf{N}=\mathbf{3 1 9}) \\
\end{array}$ & $P$ value \\
\hline $\mathrm{CPB}(\min )$ & $60(49-80)$ & $85(66-113)$ & $<.001$ \\
\hline Crossclamp (min) & $46(37-58)$ & $68(53-92)$ & $<.001$ \\
\hline ICU LOS (d) & $2(1-6)$ & $2(1-4)$ & .460 \\
\hline ICU reintubation & $5(7 \%)$ & $17(5 \%)$ & .664 \\
\hline ICU readmission & $3(4 \%)$ & $21(7 \%)$ & .381 \\
\hline Hospitalization time (d) & $10(7-15)$ & $9(7-14)$ & .538 \\
\hline Prolonged LOS $>14 \mathrm{~d}$ & $20(26 \%)$ & $77(24 \%)$ & .702 \\
\hline Short LOS $<6 \mathrm{~d}$ & $18(24 \%)$ & $75(24 \%)$ & .985 \\
\hline $\mathrm{RBC}$ transfusions & $54(71 \%)$ & $229(72 \%)$ & .805 \\
\hline $\begin{array}{l}\text { Readmission postdischarge } \\
\text { home }\end{array}$ & $5(7 \%)$ & $30(9 \%)$ & .368 \\
\hline \multicolumn{4}{|l|}{ Early complications $<30 \mathrm{~d}$} \\
\hline Mortality $<30 \mathrm{~d}$ (causes) & $4(5 \%)$ & $19(6 \%)$ & .817 \\
\hline Low cardiac output & $2(50 \%)$ & $6(32 \%)$ & .343 \\
\hline $\begin{array}{l}\text { Stroke with permanent } \\
\text { deficit }\end{array}$ & $0(0 \%)$ & $1(5 \%)$ & .272 \\
\hline Infection sepsis & $2(50 \%)$ & $7(37 \%)$ & .144 \\
\hline Multiple organ failure & $0(0 \%)$ & $5(26 \%)$ & .396 \\
\hline Acute kidney injury & $32(42 \%)$ & $109(34 \%)$ & .194 \\
\hline Atrial fibrillation & $33(43 \%)$ & $158(50 \%)$ & .338 \\
\hline Pacemaker implantation & $13(17 \%)$ & $26(8 \%)$ & .019 \\
\hline $\begin{array}{l}\text { Renal failure requiring } \\
\text { temporary dialysis } \\
\text { CVVH }\end{array}$ & $10(13 \%)$ & $27(8 \%)$ & .207 \\
\hline $\begin{array}{l}\text { Tamponade, reoperation for } \\
\text { bleeding }\end{array}$ & $6(8 \%)$ & $27(8 \%)$ & .872 \\
\hline $\begin{array}{l}\text { Any cerebrovascular } \\
\text { accident }\end{array}$ & $3(4 \%)$ & $14(4 \%)$ & .865 \\
\hline Myocardial infarction & $0(0 \%)$ & $0(0 \%)$ & 1.000 \\
\hline Paravalvular leak & $0(0 \%)$ & $0(0 \%)$ & 1.000 \\
\hline
\end{tabular}

Stroke is with or without sequelae/thromboembolic events: deep vein thrombosis and pulmonary embolism; reexploration for bleeding is hemorrhage and tamponade. AVR, Aortic valve replacement; $C P B$, cardiopulmonary bypass; $I C U$, intensive care unit; $L O S$, length of stay; $R B C$, red blood cell; $C V V H$, continuous venovenous hemofiltration.

$1.5 \pm 0.4 \mathrm{~cm}^{2}$. Fourteen patients $(14 / 76,18 \%)$ underwent implantation of a small-sized Perceval prosthesis, and 25 patients $(25 / 319,8 \%)$ had a $19-\mathrm{mm}$ or $21-\mathrm{mm} \mathrm{SV}$ prosthesis.

TABLE 4. Pre and post mean gradient $(\mathrm{mm} \mathrm{Hg})$ and aortic area $\left(\mathrm{cm}^{2}\right)$

\begin{tabular}{|c|c|c|c|}
\hline & $\begin{array}{c}\text { Sutureless } \\
\text { valve } \\
(\mathbf{N}=76) \\
\end{array}$ & $\begin{array}{c}\text { Stented } \\
\text { biological AVR } \\
(\mathbf{N}=\mathbf{3 1 9}) \\
\end{array}$ & $P$ value \\
\hline $\begin{array}{l}\text { Preoperative mean gradient } \\
\quad(\mathrm{mm} \mathrm{Hg})\end{array}$ & $44.5 \pm 16.6$ & $46.3 \pm 13.3$ & .5 \\
\hline Preoperative aortic area $\left(\mathrm{cm}^{2}\right)$ & $0.8 \pm 0.3$ & $0.7 \pm 0.2$ & .6 \\
\hline $\begin{array}{l}\text { Postoperative mean gradient } \\
\quad(\mathrm{mm} \mathrm{Hg})\end{array}$ & $15.5 \pm 7.3$ & $14.6 \pm 4.7$ & .5 \\
\hline $\begin{array}{l}\text { Postoperative aortic } \\
\text { area }\left(\mathrm{cm}^{2}\right)\end{array}$ & $1.5 \pm 0.5$ & $1.5 \pm 0.4$ & .8 \\
\hline
\end{tabular}


TABLE 5. Perioperative outcomes in subgroup analysis patients with aortic valve replacement and coronary artery bypass grafting

\begin{tabular}{lccr}
\hline & \begin{tabular}{c} 
Sutureless \\
valve \\
\multicolumn{1}{c}{ Outcomes }
\end{tabular} & $\begin{array}{c}\text { Stented } \\
\text { biological AVR }\end{array}$ & \\
& $(\mathbf{N}=\mathbf{3 2})$ & $\mathbf{( N = 1 6 8 )}$ & $\boldsymbol{P}$ value \\
\hline CPB (min) & $67(61-81)$ & $106(88-127)$ & $<.001$ \\
Crossclamp (min) & $53(50-67)$ & $88(69-102)$ & $<.001$ \\
Time spent in surgery & $148(133-172)$ & $198(165-235)$ & $<.001$ \\
ICU LOS (d) & $2(1-5)$ & $3(1-4)$ & .345 \\
Hospitalization time (d) & $11(7-14)$ & $8(6-15)$ & .571 \\
RBC transfusions & $25(78 \%)$ & $128(77 \%)$ & .936 \\
Early complications <30 d & & & \\
$\quad$ Mortality <30 d (causes) & $1(3 \%)$ & $8(5 \%)$ & .602 \\
Stroke with permanent & $0(0 \%)$ & $2(1 \%)$ & .376 \\
$\quad$ deficit & & & \\
Acute kidney injury & $11(34 \%)$ & $58(35 \%)$ & .506 \\
Atrial fibrillation & $13(41 \%)$ & $77(46 \%)$ & .376 \\
Pacemaker implantation & $4(13 \%)$ & $13(8 \%)$ & .061 \\
Paravalvular leak & $0(0 \%)$ & $0(0 \%)$ & 1.000 \\
\hline
\end{tabular}

$A V R$, Aortic valve replacement; $C P B$, cardiopulmonary bypass; $I C U$, intensive care unit; $L O S$, length of stay; $R B C$, red blood cell.

\section{Subgroup Analyses}

Isolated aortic valve replacement. A total of 43 patients underwent isolated AVR with the Perceval prosthesis (Table 1). Thirty-day mortality was similar in the 2 groups $(3 / 43[7 \%]$ in the Perceval group and $10 / 151[7 \%]$ in the
SV group). Minimally invasive approaches (mini-sternotomy or thoracotomy) were used in 14 patients $(14 / 43$, $33 \%$ ) with the Perceval valve compared with 24 patients $(24 / 151,16 \%)$ with the SV $(P=.103)$. The 30 -day mortality of patients who received the minimally invasive access procedure was $5 \%$ versus $7 \%$ for standard sternotomy $(P=.94)$. The time in surgery was shorter: 110 minutes (IQR, 98-147) versus 142 minutes (IQR, 117-161) $(P<.001)$. Bypass and crossclamp times also were lower in the minimally invasive approach group: 53 minutes (IQR, 44-66) $P<.001$ and 41 minutes (IQR, 35-48) $P<.001$, respectively, compared with 71 minutes (IQR, 57-85) $P<.001$ and 56 minutes (IQR, 43-66) $P<.001$, respectively, in the standard sternotomy group. There were no differences in all other perioperative outcomes.

Combined aortic valve replacement and coronary artery bypass grafting. euroSCORE II and STS scores, and demographics were similar in the 2 groups (Table 5). Mortality at 30 days was 3\% (1/32 patients) in the Perceval group with CABG and 5\% $(8 / 168,5 \%)$ in the SV group with CABG $(P=.6)$. There was no difference in any morbidities (low cardiac output syndrome, reexploration for tamponade, acute kidney injury with or without dialysis, sepsis, pulmonary complication, multiorgan failure) $(P=.4)$. ICU and hospital length of stay, and number of transfusions received were similar. The rate of pacemaker

TABLE 6. Propensity score matching (1:2): Demographics

\begin{tabular}{|c|c|c|c|}
\hline Demographics & $\begin{array}{c}\text { Sutureless valve } \\
\text { (65) }\end{array}$ & $\begin{array}{c}\text { Stented biological AVR } \\
\text { (130) }\end{array}$ & Standardized difference \\
\hline Age (y) & $83 \pm 3$ & $83 \pm 3$ & -15.7 \\
\hline Sex (male) & 52 & 61 & -19.5 \\
\hline BMI & $28 \pm 4$ & $27 \pm 5$ & 19.8 \\
\hline Hypertension (\%) & 86 & 83 & 8.5 \\
\hline Diabetes & 36 & 37 & -2.8 \\
\hline Preoperative eGRF & $51(38-65)$ & $56(41-71)$ & -14.5 \\
\hline Peripheral vascular disease & $3(4.1)$ & $2(2.7)$ & 7.5 \\
\hline Any prior neurologic event & $7(9.6)$ & $10(13.7)$ & -12.8 \\
\hline Chronic obstructive pulmonary disease & $13(20.0)$ & $21(16.2)$ & 10.0 \\
\hline Ever smoker & $3(4.6)$ & $7(5.4)$ & -3.7 \\
\hline Prior cardiac surgery & $4(6.2)$ & $4(3.1)$ & 14.7 \\
\hline Preoperative NYHA class III or IV & $30(46.2)$ & $63(48.5)$ & -4.6 \\
\hline LVEF $<40 \%$ & $3(4.6)$ & $8(6.2)$ & -6.8 \\
\hline Aortic valve mean gradient & $44 \pm 17$ & $45 \pm 21$ & -8.8 \\
\hline Aortic valve area & $0.74 \pm 0.21$ & $0.77 \pm 0.21$ & -13.3 \\
\hline No. of diseased vessels, none & $36(55.4)$ & $59(45.4)$ & 26.9 \\
\hline Logistic euroSCORE II & $4.4 \%(2.8 \%-8.4 \%)$ & $5.3 \%(3.1 \%-9.0 \%)$ & -10.6 \\
\hline STS 30-d mortality & $5.6 \%(4.1 \%-8.4 \%)$ & $5.8 \%(4.4 \%-7.9 \%)$ & -2.1 \\
\hline Diuretics & $29(44.6)$ & $67(51.9)$ & -14.7 \\
\hline Surgical approach & & & 32.3 \\
\hline Aortic valve prosthesis size & & & 2.1 \\
\hline Cardiopulmonary bypass time, $\min$ & $59(48-79)$ & $85(64-107)$ & -92.0 \\
\hline Crossclamp time, $\min$ & $43(37-53)$ & $64(51-89)$ & -97.1 \\
\hline
\end{tabular}

Matched = standardized interval between -20 and 20. AVR, Aortic valve replacement; $B M I$, body mass index; $e G R F$, estimated glomerular filtration rate; $N Y H A$, New York Heart Association; $L V E F$, left ventricular ejection fraction; euroSCORE, European System for Cardiac Operative Risk Evaluation; STS, Society of Thoracic Surgeons. 
use was $13 \%$ in the Perceval group and $8 \%$ in the SV group. Cardiopulmonary bypass and aortic crossclamp times were lower in the Perceval group (67 minutes [IQR, 61-81] and 53 minutes [IQR, 50-67] compared with the SV group 106 minutes [IQR, 88-127] and 88 minutes [IQR, 69102]) $(P<.001)$. The time spent in surgery was decreased in the Perceval group compared with standard AVR group (148 minutes [IQR, 133-172] and 198 minutes [IQR, 165235]) $(P<.001)$ (Table 5).

Propensity-matched group: Perceval versus standard prostheses. Sixty-five patients in the Perceval group were matched to 130 patients in the stented prosthesis group (Table 6). Bypass and crossclamp times were lower in the Perceval group: 59 minutes (IQR, 48-79) and 43 minutes (IQR, 37-53), respectively, versus 85 minutes (IQR, 64-107) and 64 minutes (IQR, 51-89) in the SV group, respectively (Table 6). There were no differences in perioperative outcomes. Pacemaker rates were similar in the 2 groups, and 30-day mortality was similar (Table 7).

\section{DISCUSSION}

The present study suggests that the sutureless prosthesis could be a reasonable alternative to standard AVR with a stented biological aortic valve to decrease the cardiopulmonary bypass and crossclamp times in patients aged 80 years or more. However, it did not translate into a reduction of postoperative adverse outcomes. With similar surgical risk (euroSCORE II and STS

TABLE 7. Perioperative outcomes for patients matched 1:2

\begin{tabular}{lccc}
\hline \multicolumn{1}{c}{ Perioperative outcomes } & $\begin{array}{c}\text { Sutureless } \\
\text { valve } \\
\mathbf{( 6 5 )}\end{array}$ & $\begin{array}{c}\text { Stented } \\
\text { biological AVR } \\
(\mathbf{1 3 0})\end{array}$ & $\boldsymbol{P}$ value \\
\hline 30-d Mortality & $4(6.2)$ & $10(7.7)$ & .70 \\
Prolonged ventilation $(>24 \mathrm{~h})$ & $4(6.2)$ & $5(4.1)$ & .56 \\
ICU LOS (first stay), d & $2.0(1.0-6)$ & $2.0(1.0-4)$ & .65 \\
Reintubation & $5(7.8)$ & $6(4.7)$ & .39 \\
Readmission to ICU & $3(4.6)$ & $8(6.2)$ & .65 \\
Any transfusion & $46(70.8)$ & $93(71.5)$ & .92 \\
Any cardiac reoperation & $7(10.8)$ & $10(7.7)$ & .49 \\
Hospital LOS (d) & $10(6-15)$ & $8(7-13)$ & .33 \\
Prolonged LOS (>14 d) & $18(27.7)$ & $26(20.0)$ & .26 \\
Renal failure & $9(13.8)$ & $11(8.5)$ & .28 \\
Acute kidney injury & $30(46.2)$ & $46(35.4)$ & .14 \\
$\quad$ (delta creatinine & & & \\
$\quad>100 \mu$ mol/L or $>50 \%)$ & & & .44 \\
Any cerebrovascular accident & $2(3.1)$ & $7(5.4)$ & .32 \\
Postoperative myocardial & $0(0)$ & $1(0.8)$ & \\
$\quad$ infarction & & & .36 \\
Atrial fibrillation & $27(41.5)$ & $63(48.5)$ & .33 \\
New pacemaker implantation & $10(15.4)$ & $13(10.0)$ & .15 \\
Multiple organ failure & $0(0)$ & $2(1.5)$ & .17 \\
Any 30-d morbidity & $55(84.6)$ & $99(76.2)$ & .51 \\
Destination at discharge & & &
\end{tabular}

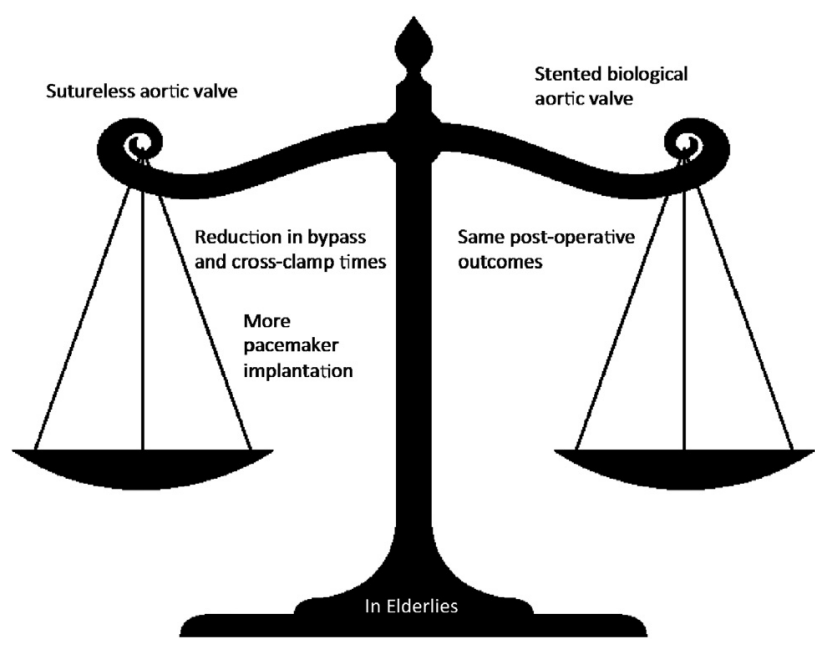

FIGURE 1. We compared sutureless and stented biologic aortic valves in elderly patients.

score), we did not find significant differences in mortality and major morbidities between the 2 groups except for a higher rate of pacemaker implantation in the Perceval group. We studied a subgroup of patients who underwent AVR with concomitant CABG procedures and found a lower 30-day mortality in those who received the Perceval prosthesis, but this difference was not statistically significant (Figure 1).

Few studies have reported the rate of pacemaker implantation after sutureless AVR, but Santarpino and colleagues ${ }^{4}$ showed a rate of $6 \%$ in their study. Our rate of pacemaker implantation is lower than what is reported with the transapical valve, such as the CoreValve (Medtronic Inc) $(33 \%){ }^{12}$ but similar to rates with the Edwards Lifesciences SAPIEN valve $(15 \%-20 \%){ }^{13}$

Nine of 13 patients in our study had a significant history of preoperative arrhythmia. The valve is made of a nitinol frame and descends down the left ventricular outflow tract. It could result in an increase in pressure on the membranous septum as the prosthesis expands and cause AV block, especially in patients with a history of bundle branch block. Sorin Group (Milan, Italy) recommends inflating the balloon at $4 \mathrm{~atm}$ for all valve sizes. It is different than what is seen with the transcatheter AVR prosthesis with a smaller volume inflation with a smaller prosthesis. This also might contribute to the high number of AV blocks. Supra-annular placement should be an alternative, but we need to be sure that there is enough calcium to anchor the valve. A less-aggressive decalcification of the annulus could be a way to avoid AV block, but may expose the patient to a greater risk of paravalvular leak. Several groups have reported reoperation for paravalvular leak $(3 \%)$ as early as 13 days. ${ }^{7,14}$ One case report also showed patientprosthesis mismatch in a patient who had a sutureless valve implanted. ${ }^{15}$ We did not encounter these types of 
complications in our cohort of patients who had a Perceval valve or in patients with a standard AVR, but our follow-up remains short.

A study by Folliguet and colleagues ${ }^{7}$ in 2012 reported the midterm results of a prospective, multicenter clinical study. They evaluated the safety and efficacy of the Perceval S valve in 208 patients undergoing AVR with or without concomitant procedures. The mean follow-up time was $10 \pm 20$ months. The mean preoperative and postoperative gradients were $48.6 \pm 18.6 \mathrm{~mm} \mathrm{Hg}$ and $10.4 \pm 4.3 \mathrm{~mm} \mathrm{Hg}$, respectively, and preoperative and postoperative mean effective orifice areas were $0.7 \pm 0.2 \mathrm{~cm}^{2}$ and $1.4 \pm 0.4 \mathrm{~cm}^{2}$. During follow-up, 9 patients $(4 \%)$ required reoperation for paravalvular regurgitation: 7 early and 2 late reoperations. ${ }^{7}$ This shows good long-term hemodynamic results and a small percentage of reoperation for paravalvular leak.

In 2014, Santarpino and colleagues ${ }^{16}$ published a propensity-matched study comparing the clinical and echocardiographic outcomes between patients undergoing transcatheter aortic valve implantation (TAVI) and sutureless AVR. Their results showed that in-hospital mortality was similar between the groups $(P=.24)$, as well as pacemaker implantation $(P=.18)$ and neurologic events. However, predischarge echocardiographic data showed a higher paravalvular leak rate in the TAVI group: $13.5 \%$ versus $0 \%$ $(P=.027)$. At a mean follow-up of $18.9 \pm 10.1$ months, overall cumulative survival was $91.9 \%$ and significantly differed between groups: sutureless $97.3 \%$ versus TAVI $86.5 \%(P=.015) .{ }^{16}$ A group from the Mayo Clinic recently published their experience with surgical or transfemoral AVR using the SAPIEN transcatheter heart valve in nonagenarians. Operative mortality was similar, but there was an increased risk of vascular injury in the group treated with TAVI. In this case, the Perceval valve could be an alternative to a less-extensive surgery, although we were not able to prove in this article that shorter bypass and crossclamp times resulted in less postoperative adverse outcomes. ${ }^{17}$ Gilmanov and colleagues ${ }^{18}$ also showed decreased bypass and crossclamp times. However, this did not translate into decreased hospital mortality or incidence of postoperative complications except for a shorter duration of mechanical ventilation in the Perceval group. ${ }^{18,19}$ Further studies need to be performed to construct a proper algorithm for patients in the "gray zone" among the conventional surgical AVR, sutureless AVR, and TAVI approaches.

In our study, $19 \%$ of patients had a sutureless valve implanted by mini-sternotomy or thoracotomy versus $8 \%$ in the SV group. Surgeons found that sutureless valves are easier to implant and more suitable for a minimally invasive approach compared with the standard stented prosthesis. Borger and colleagues ${ }^{19}$ recently published a randomized multicenter trial comparing the outcomes of a sutureless aortic valve implanted by a minimally invasive approach with a standard AVR by full sternotomy. They concluded that the minimally invasive approach with the sutureless valve resulted in significantly reduced ischemic time and better hemodynamic function in small annuli with no adverse clinical outcomes. ${ }^{19}$

Other complications related to AVR, such as perioperative thromboembolic events, myocardial infarction, reexploration for bleeding, and renal failure requiring dialysis, were comparable to those published in the literature. ${ }^{5,7}$ Recent results by 2 European centers including 281 patients who underwent AVR with the Perceval $S$ valve showed that the overall survival at a median follow-up of 8 months was $90 \%$ with in-hospital mortality of $0.7 \%$ and fewer postoperative complications. ${ }^{20}$ This sutureless valve is a proven technology with short-term follow-up and could now be included in our armamentarium for the treatment of aortic valve disease. However, longer followup and larger trials are needed to discriminate which specific population will benefit the most from this valve.

Bypass time and crossclamp times were shorter in the Perceval group, and this could lead to fewer transfusions and shorter ICU, intubation, and hospitalization times, as suggested by several studies. ${ }^{1,3,4}$ In the current experience, we did not find significant differences in these clinical parameters, although performing an AVR with the Perceval prosthesis resulted in a shorter procedural time with no increased risk of complications. Moreover, there are certainly nonmeasured variables that could be different in the 2 groups, such as the Folstein test, the 5-m walk test, and some other frailty parameters that could not be assessed because of the retrospective aspect of this study.

The appropriate surgical indications for the use of sutureless prostheses are in progress. Minimally invasive approaches and heavily calcified and small aortic roots in elderly but operable patients remain significant technical challenges that could be addressed with these newer prostheses as opposed to standard sutured prostheses. Although sutureless prostheses are not an alternative to transcatheter AVR, it will become an interesting option for high-risk and elderly patients who require concomitant $\mathrm{CABG}$ procedures with immediate postimplantation results in term of absence of paravalvular leak.

\section{Study Limitations}

This study is retrospective and may have a selection bias because the choice of the prosthesis was left to the patients and surgeons.

\section{CONCLUSIONS}

AVR with the sutureless Perceval prosthesis resulted in shorter aortic crossclamp and bypass times compared with the stented biological valve. The reduced cardiopulmonary bypass and aortic crossclamp times obtained using the 
Perceval prosthesis did not translate into perioperative gains in our elderly patient population.

\section{Conflict of Interest Statement}

Philippe Demers reports lecture fees from Sorin. All other authors have nothing to disclose with regard to commercial support.

\section{References}

1. Bacchetta MD, Ko W, Girardi LN, Mack CA, Krieger KH, Isom OW, et al. Outcomes of cardiac surgery in nonagenarians: a 10-year experience. Ann Thorac Surg. 2003;75:1215-20.

2. Hovanesyan A, Moon MR, Rich MW. Cardiac surgery in nonagenarians. J Cardiovasc Surg (Torino). 2007;48:757-60.

3. Ullery BW, Peterson JC, Milla F, Wells MT, Briggs W, Girardi LN, et al. Cardiac surgery in select nonagenarians: should we or shouldn't we? Ann Thorac Surg. 2008;85:854-60.

4. Santarpino G, Pfeiffer S, Concistre G, Grossmann I, Hinzmann M, Fischlein T. The Perceval S aortic valve has the potential of shortening surgical time: does it also result in improved outcome? Ann Thorac Surg. 2013;96:77-82.

5. Santarpino G, Pfeiffer S, Schmidt J, Concistre G, Fischlein T. Sutureless aortic valve replacement: first-year single-center experience. Ann Thorac Surg. 2012; 94:509.

6. Flameng W, Herregods MC, Hermans H, Van der Mieren G, Vercalsteren M, Poortmans G, et al. Effect of sutureless implantation of the Perceval S aortic valve bioprosthesis on intraoperative and early postoperative outcomes. J Thorac Cardiovasc Surg. 2011;142:1453-7.

7. Folliguet TA, Laborde F, Zannis K, Ghorayeb G, Haverich A, Shrestha M. Sutureless Perceval aortic valve replacement: results of two European centers. Ann Thorac Surg. 2012;93:1483-8.

8. Shrestha M, Maeding I, Hoffler K, Koigeldiyev N, Marsch G, Siemeni T, et al. Aortic valve replacement in geriatric patients with small aortic roots: are sutureless valves the future? Interact Cardiovasc Thorac Surg. 2013;17:778-82.

9. Phan K, Tsai YC, Niranjan N, Bouchard D, Carrel TP, Dapunt OE, et al. Sutureless aortic valve replacement: a systematic review and meta-analysis. Ann Cardiothorac Surg. 2015;4:100-11.
10. Akins CW, Miller DC, Turina MI, Kouchoukos NT, Blackstone EH, Grunkemeier GL, et al. Guidelines for reporting mortality and morbidity after cardiac valve interventions. J Thorac Cardiovasc Surg. 2008;135:732-8.

11. Levey AS, Stevens LA, Schmid CH, Zhang YL, Castro AF III, Feldman HI, et al. A new equation to estimate glomerular filtration rate. Ann Intern Med. 2009;150: 604-12.

12. Khawaja MZ, Rajani R, Cook A, Khavandi A, Moynagh A, Chowdhary S, et al. Permanent pacemaker insertion after CoreValve transcatheter aortic valve implantation: incidence and contributing factors (the UK CoreValve Collaborative). Circulation. 2011;123:951-60.

13. Thomas M, Schymik G, Walther T, Himbert D, Lefevre T, Treede H, et al. Thirtyday results of the SAPIEN aortic Bioprosthesis European Outcome (SOURCE) Registry: a European registry of transcatheter aortic valve implantation using the Edwards SAPIEN valve. Circulation. 2010;122:62-9.

14. Shrestha M, Folliguet T, Meuris B, Dibie A, Bara C, Herregods MC, et al. Sutureless Perceval $\mathrm{S}$ aortic valve replacement: a multicenter, prospective pilot trial. $J$ Heart Valve Dis. 2009;18:698-702.

15. Hajj-Chahine J. eComment. Incidence of patient-prosthesis mismatch in patients with a Perceval S valve. Interact Cardiovasc Thorac Surg. 2013;17:782-3.

16. Santarpino G, Pfeiffer S, Jessl J, Dell'aquila AM, Pollari F, Pauschinger M, et al. Sutureless replacement versus transcatheter valve implantation in aortic valve stenosis: a propensity-matched analysis of 2 strategies in high-risk patients. $J$ Thorac Cardiovasc Surg. 2014;147:561-7.

17. Murashita T, Greason KL, Suri RM, Nkomo VT, Holmes DR, Rihal CS, et al. Aortic valve replacement for severe aortic valve stenosis in the nonagenarian patient. Ann Thorac Surg. 2014;98:1593-7.

18. Gilmanov D, Miceli A, Ferrarini M, Farneti P, Murzi M, Solinas M, et al. Aortic valve replacement through right anterior minithoracotomy: can sutureless technology improve clinical outcomes? Ann Thorac Surg. 2014;98:1585-92.

19. Borger MA, Moustafine V, Conradi L, Knosalla C, Richter M, Merk DR, et al. A randomized multicenter trial of minimally invasive rapid deployment versus conventional full sternotomy aortic valve replacement. Ann Thorac Surg. 2015;99: $17-25$.

20. Miceli A, Santarpino G, Pfeiffer S, Murzi M, Gilmanov D, Concistre G, et al. Minimally invasive aortic valve replacement with Perceval S sutureless valve: Early outcomes and one-year survival from two European centers. J Thorac Cardiovasc Surg. 2014;148:2838-43.

Key Words: sutureless aortic valve replacement 